\title{
Effect of propranolol on the QT intervals of normal individuals during exercise: a new method for studying interventions
}

\author{
JONNALAGEDDA S M SARMA, K VENKATARAMAN, DINESH R SAMANT, \\ UDAY G GADGIL \\ From the Department of Cardiology, City of Hope National Medical Center, Duarte, California, USA
}

SUMMARY A new method was used to study the effect of a single dose of propranolol on the QT intervals during exercise in 11 normal volunteers. They exercised maximally on a bicycle ergometer and repeated the test after taking propranolol $(40 \mathrm{mg})$ by mouth two hours before. Electrocardiograms were continuously recorded on magnetic tape and the cardiac cycle length (RR interval) and the QT interval were measured every five seconds by a computer aided method. The RR-QT data from each test during the exercise phase were analysed by an exponential formula, QT $=A-B \times \exp (-k \times R R)$ and by Bazett's formula, $Q T=K \times \sqrt{ }(\mathbf{R} R)$. Three reference QT intervals, $Q T_{c 1}, Q T_{c 2}$, and $Q T_{c 3}$, estimated at $R R=400,700$, and $1000 \mathrm{~ms}$ respectively from the regression curves of both formulas were compared. The exponential formula, which consistently gave a better fit with the data, showed that propranolol had a biphasic action on the QT intervals during exercise. It significantly prolonged the mean (SD) interval at longer cycle lengths (from 287 (27) to 305 (18) $\mathrm{ms}$ at $\mathrm{RR}=1000 \mathrm{~ms}$ and shortened it at shorter cycle lengths (from 198 (14) to 179

(16) $\mathrm{ms}$ at $R R=400 \mathrm{~ms}$ ). In contrast, Bazett's formula did not show any significant effect when the same raw data were used.

The exponential formula can be adapted to study other interventions or conditions that affect QT intervals.

The results of previous studies of the effect of propranolol on the QT interval are contradictory. Milne et al showed that intravenous propranolol prolonged the QT interval at identical atrial paced cycle lengths, ${ }^{1}$ whereas Browne et al found no significant changes under similar conditions'; however, the use of Bazett's formula ${ }^{34}$ shortened the rate corrected QT interval calculated from both studies. Other studies of propranolol also suggested that the QT interval corrected for heart rate by Bazett's formula was usually shortened. ${ }^{36}$

We found that the functional dependence of the human QT interval on the cardiac cycle length ( $R R$ interval) during exercise and cardiac pacing was well expressed by an exponential formula. ${ }^{7}$ We used this exponential formula to evaluate the effects of

Requests for reprints to Dr $K$ Venkataraman, Department of Cardiology, City of Hope National Medical Center, 1500 East Duarte Road, Duarte, CA 91010, USA.

Accepted for publication 13 June 1988 propranolol on the QT intervals of normal individuals during exercise and we compared the results with an analysis of the same data by Bazett's formula.

\section{Patients and methods}

Eleven healthy volunteers (six men and five women, mean (SD) age 34 (7) years (range 20-48)) exercised maximally on a bicycle ergometer. They exercised once without medication after an overnight fast, and seven to 10 days later they exercised again after taking $\mathbf{4 0} \mathrm{mg}$ of propranolol by mouth two to three hours before the test. This dose slowed the heart rate and caused visible symptoms of tiredness in all the volunteers, none of whom had taken propranolol before. For each test, the initial exercise load was set at $50 \mathrm{~W}$ and was increased in steps of $25 \mathrm{~W}$ every two minutes. Electrocardiographic leads I, aVF, and V5 were continuously recorded on an analogue tape recorder (Model HP3964A, Hewlett-Packard, Palo 
Alto, CA) that was started three minutes before exercise. This study protocol was approved by our institutional review board. Each person signed a form giving informed consent.

The $R R$ and QT intervals were measured automatically every five seconds by a programmable waveform analyser (Model 3001, Norland, Fort Atkinson, WI) and the data were transmitted to an IBM-PC/AT microcomputer (IBM Personal Computer Division, Boca Raton, FL) over an IEEE-488 interface. The QT interval was measured from the beginning of the $Q R S$ complex to the apex of the $T$ wave. In eight people in whom the end of the $T$ wave could be clearly identified throughout the exercise the average interval from apex $T$ to end $T$ (eT-aT interval) was measured at rest and peak exercise. The end of the $T$ wave was taken as the point at which the line drawn through the steepest portion of the terminal limb of the $T$ wave cut the isoelectric line defined by the $P Q$ segment. ${ }^{8}$

The RR-QT data during the exercise phase were fitted to the exponential formula: $Q T=A-B$ $\times \exp (-k \times R R)$, where the parameters $A, B$, and $k$ were estimated by non-linear regression with the ASYST scientific system (Macmillan Software, New York, NY). For comparison, the exercise RRQT plots were also fitted to Bazett's formula ${ }^{3}$ : $\mathbf{Q T}=\mathbf{K} \times \sqrt{ }(\mathbf{R R})$, in which the parameter $\mathbf{K}$ was estimated by linear regression. The measurements made during the recovery phase were not included in the analysis, to avoid the effects caused by QT hysteresis.

\section{DATA ANALYSIS}

We compared the three reference $Q T$ intervals, $Q T_{c 1}$, $\mathrm{QT}_{\infty}$, and $\mathrm{QT}_{\mathrm{c3}}$, estimated from the individual regression curves at $R R=400,700$, and $1000 \mathrm{~ms}$, respectively, in the control and propranolol groups. These estimates may be regarded as corrected QT intervals (see the Appendix). They are expressed as functions of the regression equations as follows:

$$
\begin{aligned}
& \mathrm{QT}_{\mathrm{cl}}=\mathrm{A}-\mathrm{B} \times \operatorname{Exp}(-\mathrm{k} \times \quad \mathbf{4 0 0}) \\
& \mathrm{QT}_{\mathrm{c} 2}=\mathrm{A}-\mathrm{B} \times \operatorname{Exp}(-\mathrm{k} \times \mathbf{7 0 0}) \\
& \mathrm{QT}_{\mathrm{c} 3}=\mathrm{A}-\mathrm{B} \times \operatorname{Exp}(-\mathrm{k} \times 1000),
\end{aligned}
$$

where the $Q T_{c}$ intervals are expressed in ms. A set of three $Q T_{c}$ intervals are required to characterise uniquely each exponential RR-QT curve. We chose the corrected QT intervals for statistical comparisons because this permits the drug induced changes in the QT $_{c}$ estimates to be interpreted in terms of prolongation or shortening of QT intervals in the observable range of cycle lengths. Similar estimates of the $Q T_{c} s$ derived from Bazett's (or any other) formula can be compared with the values derived from the exponential formula.
The corrected QT intervals at $R R=400,700$, and $1000 \mathrm{~ms}$ according to Bazett's formula were estimated by the following equations:

$$
\begin{aligned}
& \mathrm{QT}_{\mathrm{cl}}=\mathrm{K} \times \sqrt{ }(\mathbf{4 0 0 )} \\
& \mathrm{QT}_{\mathrm{c}}=\mathrm{K} \times \sqrt{ }(\mathbf{7 0 0 )} \\
& \mathrm{QT}_{\mathrm{c3}}=\mathrm{K} \times \sqrt{(\mathbf{1 0 0 0}),}
\end{aligned}
$$

where the $\mathrm{QT}_{\mathrm{c}}$ intervals are also expressed in $\mathrm{ms}$. $\mathrm{QT}_{\mathrm{c}}$ corresponds to the conventional corrected QT interval. Zipes recently suggested that when the effects of drugs or other interventions are being evaluated the original Bazett's formula, $Q T=K$ $x \sqrt{ }(\mathbf{R R})$, where the $K$ value is derived from a regression analysis, is superior to the commonly applied formula, $Q T_{c}=Q T / \sqrt{ }(R R) .^{9}$ The relation between the two formulas is explained in the Appendix.

We compared matched sets of control and propranolol treatment values of $Q T_{c 1}, Q_{c}$, and $\mathrm{QT}_{\mathrm{c}}$ by the one sample Hotelling $\mathrm{T}^{2}$ test for overall differences and differences among individual pairs of $\mathrm{QT}_{\mathrm{c}} \mathrm{s}$ by a paired $t$ test. We used the same method to analyse the corrected QT intervals obtained by Bazett's formula. BMDP Statistical Software (Los Angeles, CA) was used for the statistical analysis.

\section{Results}

Table 1 summarises the main results. Cardiac cycle lengths at rest and peak exercise were significantly prolonged after propranolol. Systolic blood pressure at rest and peak exercise was significantly reduced. There were no pronounced changes in diastolic blood pressures. As expected, none of the subjects showed any abnormalities during exercise or recovery. The QRS configuration was unchanged during exercise and between tests. The QRS dura-

\begin{tabular}{|c|c|c|c|c|c|}
\hline \multirow[b]{2}{*}{$\begin{array}{l}\text { Measured variable } \\
\text { CCL resting (ms) } \\
\text { CCL peak exercise (ms) } \\
\text { Systolic BP resting (mm Hg) } \\
\text { Distolic BP resting (mm Hg) } \\
\text { Systolic BP peak (mm Hg) } \\
\text { Diastolic BP peak (mm Hg) } \\
\text { Exercise duration (min) }_{\text {(mis }} \\
\text { QT } \\
\text { QT (exponential) (ms) } \\
\text { QT (exponential) (ms) } \\
\text { QT (exponential) (ms) } \\
\text { QT } \\
\text { QT (Bazett) (ms) } \\
\text { QT (Bazett) (ms) } \\
\text { QT (Bazett) (ms) }\end{array}$} & \multicolumn{2}{|c|}{ Control } & \multicolumn{2}{|c|}{ Propranolol } & $p$ (pair) \\
\hline & $\begin{array}{r}869 \\
334 \\
106 \\
70 \\
159 \\
75 \\
11 . \\
198 \\
257 \\
287 \\
196 \\
259 \\
309\end{array}$ & $\begin{array}{r}(151) \\
(23) \\
(11) \\
(7) \\
(19) \\
(12) \\
(2 \cdot 5) \\
(14) \\
(16) \\
(27) \\
(13) \\
(17) \\
(20)\end{array}$ & $\left\{\begin{array}{r}1174 \\
444 \\
99 \\
68 \\
141 \\
75 \\
10 \cdot \\
179 \\
271 \\
305 \\
198 \\
261 \\
312\end{array}\right.$ & $\begin{array}{r}(168) \\
(44) \\
(11) \\
(9) \\
(24) \\
(11) \\
3(2 \cdot 7) \\
(16) \\
(17) \\
(18) \\
(13) \\
(17) \\
(20)\end{array}$ & $\begin{array}{c}<0.001 \\
<0.001 \\
<0.05 \\
\text { NS } \\
<0.01 \\
\text { NS } \\
\text { NS } \\
<0.002 \\
<0.001 \\
<0.01 \\
\text { NS } \\
\text { NS } \\
\text { NS }\end{array}$ \\
\hline
\end{tabular}
tion remained less than $5 \mathrm{~ms}$ throughout the study. The average duration of exercise was not signifi-

Table 1 Summary of results (mean (SD)) obtained from 11 volunteers who exercised under control conditions and after propranolol

BP, blood pressure; CCL, cardiac cycle length. 


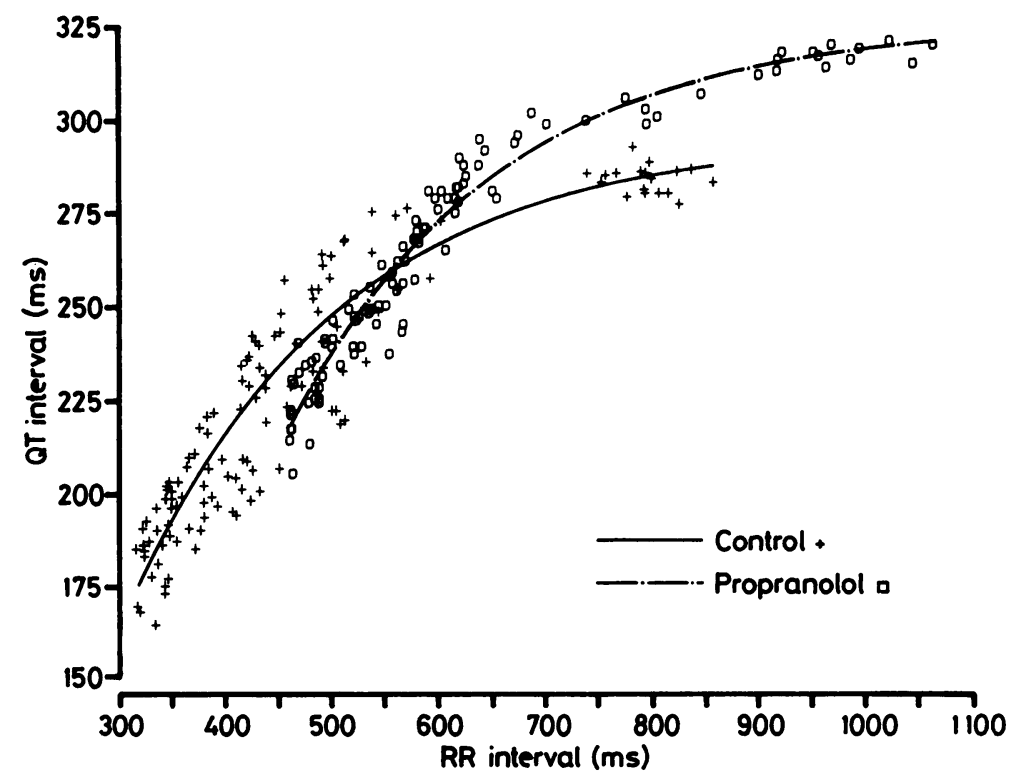

Fig 1 A typical plot showing the $R R-Q T$ relation during exercise before $(+)$ and after $(O)$ propranolol together with the fit of the regression line with the exponential formula. The apparent gap in the data points on the control curve represents a rapid shortening of cycle length during the early part of exercise. Propranolol typically caused more gradual $R R$ shortening, resulting in a more uniform distribution of data points.

cantly altered by propranolol treatment, although all the volunteers found it harder to exercise after taking the drug.

Figure 1 shows typical plots of RR versus $Q T$ and their regression lines obtained with and without propranolol treatment in one volunteer.

Table 1 shows that there were significant differences between the control and treatment values for corrected QT intervals obtained with the exponential formula $\left(Q T_{c 1}, Q_{c 2}, Q_{c 3}\right)\left(p<0.002\right.$, Hotelling $T^{2}$ test); after propranolol $Q T_{c 1}$ was significantly shortened and $\mathrm{QT}_{\mathrm{c} 2}$ and $\mathrm{QT}_{\mathrm{c}}$ were significantly prolonged. Figure 2 shows the $\mathrm{QT}_{\mathrm{c}}$ changes in individual volunteers. In contrast with the exponential formula, the corrected QT intervals obtained with Bazett's formula failed to show any significant difference between the control and treatment values (table 1), although the same raw data were used in both calculations. Figure 3 compares the curves obtained with the exponential and Bazett's formulas for the exercise data used in fig 1 . The exponential formula gave a consistently better fit with the observed data.

The changes in the $T$ wave configuration during exercise were similar under control conditions and after propranolol treatment. The average (SD) eTa $T$ intervals measured in eight volunteers at rest were
149 (17) $\mathrm{ms}$ for control and 145 (14) $\mathrm{ms}$ after propranolol $(p=N S)$. The values at peak exercise were 124 (22) for control and 125 (15) after propranolol $(p=N S)$. Thus the Q-aT and Q-eT intervals showed parallel behaviour in this study.

To assess whether the RR-QT relation is dependent on the exercise protocol, we studied a separate group of five normal volunteers (three men, two women; age 22 (5)) who exercised maximally on a bicycle ergometer ( $50 \mathrm{~W}$ initial load with $25 \mathrm{~W}$ increases every two minutes) and a treadmill (Bruce protocol) on different days in a random order. The results (table 2) show that the RR-QT relation during the exercise phase is highly reproducible and independent of the exercise protocol.

\section{Discussion}

The present study evaluated the effects of a single oral dose of propranolol $(40 \mathrm{mg})$ on the RR-QT relation in normal individuals. The effects on resting and exercise heart rate and blood pressure showed evidence of $\beta$ blockade, albeit incomplete. Under these conditions the results presented in this report clearly show a biphasic effect of propranolol on the 

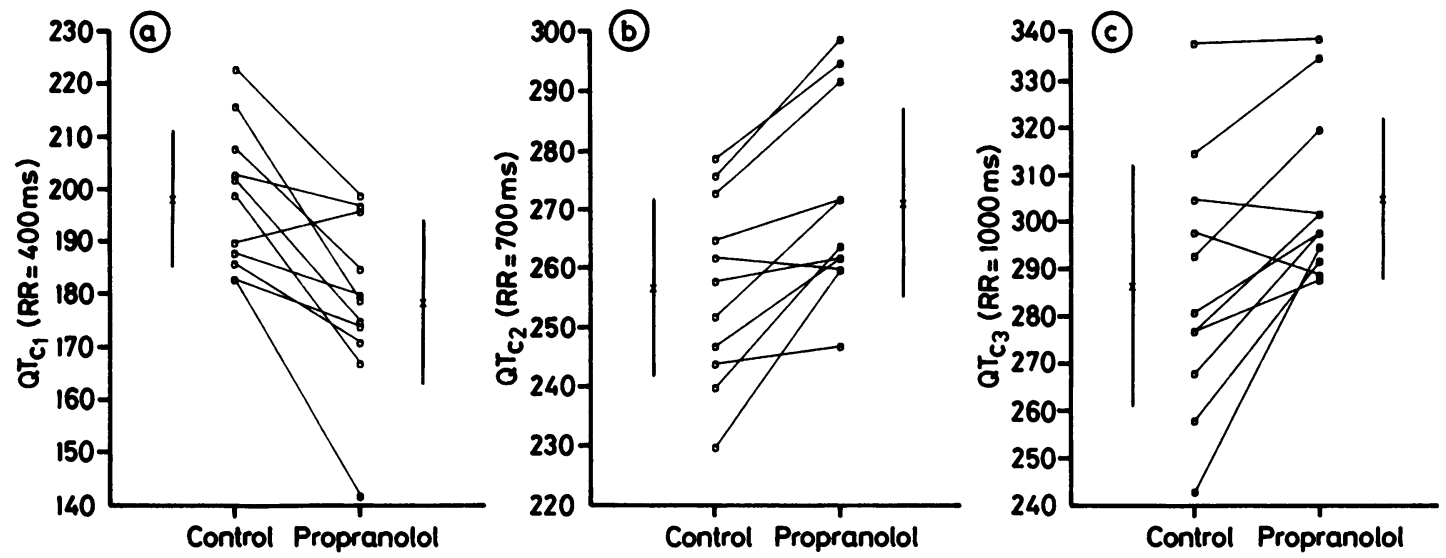

Fig 2 Effect of propranolol on the individual (a) $Q T_{\text {cl }}$ intervals estimated at $R R=400 \mathrm{~ms}$, (b) $Q T_{c 2}$ intervals estimated at $R R=700 \mathrm{~ms}$, and (c) $Q T$, intervals estimated at $R R=1000 \mathrm{~ms}$. The mean (SD) values for each group are shown. Propranolol had a biphasic effect, shortening $Q T_{c 1}(p<0.002)$ but prolonging $Q T_{c 2}(p<0.001)$ and $Q T_{c 3}(p<0.01)$.

exercise QT intervals, with net prolongation at rest and shortening at peak exercise. The high reproducibility of the exercise RR-QT curves and their apparent independence of the exercise protocol indicate the reliability of the results. Milne et al found that propranolol causes a uniform QT prolongation over a wide range of identical paced cycle lengths.' This finding, taken together with the present results, suggests that a normal tendency of propranolol to prolong the QT interval is opposed by a competing mechanism during exercise. Others have shown that pretreatment of normal individuals with propranolol increases their peak concentrations of plasma potassium and catecholamine during exercise. ${ }^{10-13}$ It was suggested that $\beta_{2}$ receptor mediated effects in the exercising muscles may cause the observed hyperkalaemia. An increased extracellular potassium concentration, which is known to shorten the action potential duration in the ventricular muscle, ${ }^{14}$ might have resulted in the relative shortening of the QT interval at peak exercise.

The measurement of QT interval to the apex of the $T$ wave rather than to its end is justified in the present study because the changes in the $T$ wave configuration during exercise did not contribute to the differences between the control and propranolol treated conditions. Under these conditions it is better to use the Q-apexT interval which can be measured more accurately. ${ }^{15}$

Propranolol is used in the treatment of long QT syndrome ${ }^{16}$ and is known to increase the otherwise low variability of the QT interval during exercise in those patients. ${ }^{17}$ It is interesting to note that propranolol also increases the responsiveness of QT intervals in normal subjects through the biphasic effect.

Though Bazett's formula is simpler to use, it is inappropriate for the study of QT intervals during exercise. Mathematically, two regression curves constructed by Bazett's formula cannot cross each other, except at the origin, irrespective of the nature of the data sets. Thus it is theoretically impossible to demonstrate the rate dependent biphasic effect of propranolol on the exercise QT interval by Bazett's formula (fig 3 ).

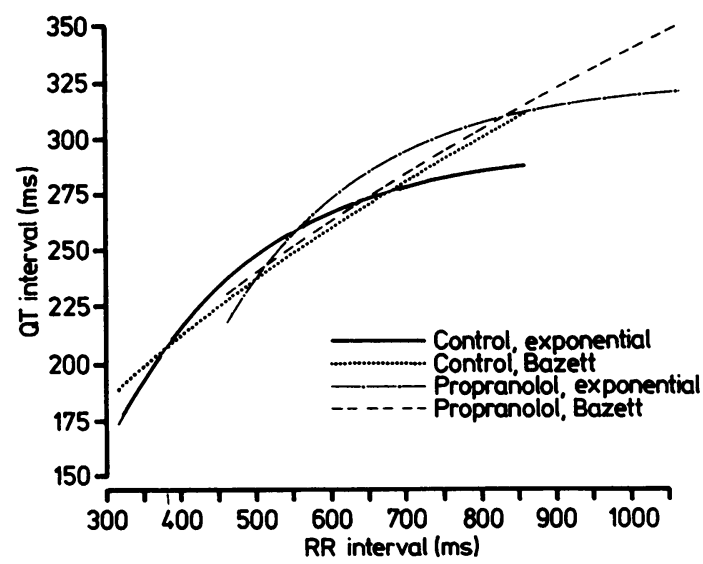

Fig 3 Comparison of the fit of the exponential and Barett formulas with the $R R-Q T$ data shown in fig 1 . The regression curves for Bazett's formula deviated markedly from the data points and showed very little change after propranolol administration. Bazett's curves are mathematically precluded from intersecting except at the origin. 
Table 2 Reproducibility of $Q T_{c}$ parameters in five normal individuals who exercised on bicycle and treadmill on different days in a random sequence

\begin{tabular}{|c|c|c|c|c|c|}
\hline No & $\begin{array}{l}\text { Exercise } \\
\text { type }\end{array}$ & $\begin{array}{l}\text { Exercise } \\
\text { duration } \\
\text { (min) }\end{array}$ & $\frac{Q T_{c l}}{(m s)}$ & $\frac{Q T_{c 2}}{(m s)}$ & $\underset{\text { (ms) }}{Q T_{c a}}$ \\
\hline 1 & Bicycle & $9 \cdot 1$ & 197 & 272 & 307 \\
\hline 2 & Bicycle. & $\begin{array}{l}14.0 \\
10.0\end{array}$ & $\begin{array}{l}198 \\
198\end{array}$ & $\begin{array}{l}269 \\
266\end{array}$ & $\begin{array}{l}309 \\
304\end{array}$ \\
\hline & Treadmill & $13 \cdot 0$ & 195 & 263 & 302 \\
\hline 3 & $\begin{array}{l}\text { Bicycle } \\
\text { Treadmill }\end{array}$ & $\begin{array}{l}10.0 \\
14.0\end{array}$ & $\begin{array}{l}189 \\
192\end{array}$ & $\begin{array}{l}256 \\
248\end{array}$ & $\begin{array}{l}285 \\
277\end{array}$ \\
\hline 4 & Bicycle & 16.0 & 199 & 266 & 295 \\
\hline 5 & $\begin{array}{l}\text { Treadmill } \\
\text { Bicycle }\end{array}$ & 12.0 & $\begin{array}{l}199 \\
188\end{array}$ & $\begin{array}{l}207 \\
252\end{array}$ & $\begin{array}{l}299 \\
281\end{array}$ \\
\hline \multirow{4}{*}{$\begin{array}{l}\text { Mean } \\
(\text { SD }) \\
\text { Mean } \\
(\text { SD) } \\
p\end{array}$} & Treadmill & $13 \cdot 4$ & 191 & 257 & 287 \\
\hline & Bicycle & $11.4(2.5)$ & $194(5)$ & $262(8)$ & 294 (11) \\
\hline & Treadmill & $13.9(0.7)$ & $195(4)$ & $260(8)$ & 294 (13) \\
\hline & & NS & NS & NS & NS \\
\hline
\end{tabular}

$Q T_{c 1}, Q T_{e s}$ and $Q T_{c 3}$ were estimated according to the exponential equations given in the text.

\section{IMPLICATIONS}

The present study offers a general method for studying the effects of various interventions on the dynamic behaviour of the QT interval. The concept of corrected QT (QT $)$ interval has been extended to be applicable to any RR-QT formula. In the case of the exponential formula, $Q T_{c}$ may be regarded as a three dimensional vector with $\mathrm{QT}_{\mathrm{cl}}, \mathrm{QT}_{\mathrm{c} 2}$, and $\mathrm{QT}_{\mathrm{c}}$ as its components. Other important features of the method include the analysis of individual RR-QT curves without prior pooling, and use of computer aided measurement of time intervals to collect accurately large numbers of data points, all of which contribute to the $\mathrm{QT}_{\mathrm{c}}$ estimates. The results presented here offer convincing evidence that the method is sensitive enough to detect subtle, but potentially important, changes in QT intervals caused by interventions. We suggest that this method should be adopted in future investigations, under appropriate conditions.

We thank our summer students Azhil Durairaj and Edward G Gillan for their help in data collection and analysis.

\section{Appendix}

\section{GENERALISED DEFINITION OF THE CORRECTED}

\section{QT $\left(Q_{\mathrm{c}}\right.$ ) INTERVAL}

To extend the concept of $Q T_{c}$ to formulas other than Bazett's, we first need to define $Q T_{c}$ in general terms as follows:

If the dependence of the QT interval on the cardiac cycle length is represented by the equation

$$
\mathbf{Q T}=\mathbf{f}(\mathbf{R R}) \text {, }
$$

where $f(R R)$ is a known function of the $R R$ interval, then $Q T_{c}$ is, by definition, given by

$$
\mathrm{QT}_{\mathrm{c}}=\mathbf{f}\left(\mathbf{R R}_{\mathrm{s}}\right) \text {, }
$$

where $R_{R_{1}}$ is a reference cycle length chosen such that $f(R R)$ gives consistent results for $R R=R R$.

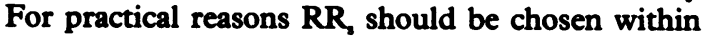
the expected physiological range of $R R$. It is possible to define more than one $\mathrm{QT}_{\mathrm{c}}$ by assigning different values to $R_{R}$. This feature is essential for the multiparameter formulas, as explained later.

\section{APPLICATION TO BAZETT'S FORMULA}

Bazett's formula is written as: $Q T=K \times \sqrt{ }(\mathbf{R R})$. Application of the above definition yields: $\mathrm{QT}_{\mathrm{c}}=$ $\mathbf{K} \times \sqrt{ }\left(\mathbf{R} \mathbf{R}_{\mathbf{\imath}}\right)$. From these two equations we get:

$$
\mathbf{K}=\frac{\mathbf{Q T}_{\mathrm{c}}}{\sqrt{ }\left(\mathbf{R} \mathbf{R}_{\mathrm{q}}\right)}=\frac{\mathbf{Q T}}{\sqrt{ }(\mathbf{R R})} .
$$

Now, if $R R$ is expressed in seconds and $R R$, is set to $1 \mathrm{~s}$, we get the popular equation:

$$
\mathrm{QT}_{\mathrm{c}}=\frac{\mathrm{QT}}{\sqrt{ }(\mathbf{R R})},
$$

which was first given by Taran and Szylagyit without a clear mathematical explanation. The general definition given above, however, is consistent with the conventional definition of $\mathrm{QT}_{\mathrm{c}}$.

\section{APPLICATION TO THE EXPONENTIAL FORMULA} According to the general definition, the $Q T_{c}$ for the exponential formula is given by:

$$
Q T_{c}=A-B \times \exp \left(-k \times R R_{\imath}\right) .
$$

Unlike Bazett's formula, however, the exponential formula requires three $\mathrm{QT}_{\mathrm{c}}$ values defined at three well spaced $R_{2}$, values, to characterise uniquely each exercise curve. These $Q T_{c}$ values may be defined as

$$
\mathrm{QT}_{\mathrm{c}}=\mathrm{A}-\mathrm{B} \times \exp \left(-\mathbf{k} \times \mathbf{R R}_{\mathrm{id}}\right) \text {, }
$$

where $R R_{\mathrm{si}}=400,700$, and $1000 \mathrm{~ms}$ for $i=1,2$, and 3 respectively. This particular set of $R R_{\mathrm{i}}$ values is suitable for comparing exercise RR-QT curves. The curves can be reconstructed from the above set of QT $T_{d}$ parameters by use of the well known NewtonRaphson recursion algorithm ${ }^{18}$ to solve for $A, B$, and k. Thus $\mathrm{QT}_{\mathrm{d}}$ and the model parameters $(\mathrm{A}, \mathrm{B}, \mathrm{k})$ contain the same information on the RR-QT curves. The former set is preferred, however, because the changes in $\mathrm{QT}_{\mathrm{c}}$ are easier to interpret in physiological terms.

In order to use multiparameter formulas, there must be some way to change the RR interval over an appropriate range to obtain reliable estimates of the model parameters. With narrow $R R$ interval ranges, the influence of measurement and rounding 
errors may render the parameter estimates unreliable. Cardiac pacing and exercise provide nonpharmacological means of producing a sufficiently wide range of $\mathbf{R R}$ intervals under fairly controlled conditions. Autonomic tests such as the Valsalva manoeuvre and cold pressor treatment may also be applied, but the $R R$ changes tend to be relatively narrow and therefore higher precision in the $R R$ and QT measurements may be required.

\section{References}

1 Milne JR, Camm AJ, Ward DE, Spurrell RAJ. Effect of intravenous propranolol on QT interval. A new method of assessment. Br Heart J 1980;43:1-6.

2 Browne KF, Zipes DP, Heger JJ, Prystowsky EN. Influence of the autonomic nervous system on the QT interval in man. Am J Cardiol 1982;50:1099-103.

3 Bazett HC. An analysis of the time relationship of electrocardiograms. Heart 1920;7:353-70.

4 Taran LM, Szylagyi N. The duration of the electrical systole (QT) in acute theumatic carditis in children. Am Heart J 1947;33:14-26.

5 Stern S, Eisenberg S. The effect of propranolol (Inderal) on the electrocardiogram of normal subjects. Am Heart J 1969;77:192-5.

6 Seides SS, Josephson ME, Batsford WP, Weisfogel GM, Lau SH, Damato AN. The electrophysiology of propranolol in man. Am Heart J 1974;88:733-41.

7 Sarma JSM, Sarma RJ, Bilitch M, Katz D, Song SL. An exponential formula for heart rate dependence of QT interval during exercise and cardiac pacing in humans. Re-evaluation of Bazett's formula. Am J Cardiol 1984;54:103-8.

8 Sarma JSM, Venkataraman K, Samant DR, Gadgil U. Hysteresis in the human RR-QT relationship during exercise and recovery. $P A C E$ 1987;102:485-91.

9 Zipes DP. Proarrhythmic effects of antiarrhythmic drugs. Am J Cardiol 1987;59:26E-31E.

10 Irving MH, Britton BJ, Wood WG, Padgham C, Carruthers $M$. Effects of beta adrenergic blockade on plasma catecholamines in exercise. Nature 1974;248: 531-3.

11 Carlsson E, Fellenius E, Lundborg P, Svensson L. Beta-adrenoceptor blockers, plasma-potassium, and exercise. Lancet 1978;ii:424-5.

12 Lim M, Linton RAF, Wolff CB, Band DM. Propranolol, exercise, and arterial plasma potassium [Letter]. Lancet 1981;il:591.

13 Williams ME, Gervino EV, Rosa RM, et al. Catecholamine modulation of rapid potassium shifts during exercise. N Engl J Med 1985;312:823-7.

14 Surawicz B. Relationship between electrocardiogram and electrolytes. Am Heart J 1967;73:814-34.

15 Hedman A, Nordlander R, Pehrsson SK. Changes in Q-T and Q-aT intervals at rest and exercise with different modes of cardiac pacing. PACE 1985;8: 825-31.

16 Schwartz PJ. Idiopathic long QT syndrome: progress and questions. Am Heart J 1985;109:399-411.

17 Raine AEG, Gribbin B, Pickering TG. Long QT syndrome: evidence for two kinds of QT prolongation [Abstract]. Br Heart J 1981;45:355.

18 Nonweiler TRF. Computational mathematics: an introduction to numerical approximation. Chicl.ester: Ellis Harwood, 1984:164. 